\title{
Intoxicação experimental com as folhas de Jatropha gossypifolia (Euphorbiaceae) em ovinos ${ }^{1}$
}

\author{
Laura I. Oliveira ${ }^{2^{\star}}$, Flávia F. Jabour ${ }^{3}$, Vivian A. Nogueira ${ }^{4}$ e Elise M. Yamasaki ${ }^{4}$ \\ ABSTRACT.- Oliveira L.I., Jabour F.F., Nogueira V.A. \& Yamasaki E.M. 2008. [Experimental \\ poisoning by the leaves of Jatropha gossypifolia (Euphorbiaceae) in sheep.] Intoxicação \\ experimental com as folhas de Jatropha gossypifolia (Euphorbiaceae) em ovinos. Pesquisa \\ Veterinária Brasileira 28(6):275-278. Projeto Sanidade Animal Embrapa/UFRRJ, Seropédica, \\ RJ 23890-000, Brazil. E-mail: iglesias.vet@gmail.com \\ Fresh green leaves of Jatropha gossypifolia (Euphorbiaceae) were lethal for sheep in \\ single administrations of $40 \mathrm{~g} / \mathrm{kg}$. The dose of $5 \mathrm{~g} / \mathrm{kg}$ did not cause poisoning, but \\ intermediate doses caused death in part of the animals. The clinical course of poisoning \\ was 6 to 22 days. The clinic and pathological picture in the experimental sheep was \\ characterized by digestive, lung and heart disturbances, and also by slight microscopic \\ liver and renal regressive alterations. These findings are similar to those observed in \\ experiments with the seeds of Jatropha curcas in goats, sheep and calves, and with the \\ fruits and leaves of Jatropha glauca and Jatropha aceroides in goats, performed by other \\ authors. A comparison indicates that, independently of the plant species, the leaves of \\ Jatropha spp. contain toxic compounds similar to those found in the seeds.
}

INDEX TERMS: Poisonous plants, plant poisoning, Jatropha gossypifolia, Euphorbiaceae, sheep, pathology.

RESUMO.- As folhas recém-colhidas de Jatropha gossypifolia (Euphorbiaceae) foram letais para ovinos em administrações únicas de $40 \mathrm{~g} / \mathrm{kg}$. A dose de $5 \mathrm{~g} / \mathrm{kg}$ não causou sintomas de intoxicação; as doses intermediárias provocaram a morte de parte dos animais. A evolução da intoxicação foi de 6 a 22 dias. O quadro clínico-patológico nos ovinos experimentais era caracterizado por perturbações digestivas, pulmonares, cardíacas e ainda alterações regressivas leves somente evidenciadas através de exames histológicos, hepáticas e renais. Esses achados são semelhantes aos observados em experimentos com as

\footnotetext{
${ }^{1}$ Recebido em 4 de dezembro de 2007.

Aceito para publicação em 22 de janeiro de 2008.

${ }^{2}$ Departamento de Epidemiologia e Saúde Pública (DESP), Universidade Federal Rural do Rio de Janeiro (UFRRJ), Seropédica, RJ 23890000, e Universidade Estácio de Sá, Rio de Janeiro, RJ, Brasil. *Autor para correspondência: iglesias.vet@gmail.com

${ }^{3}$ Departamento de Medicina Veterinária, Fundação Educacional Jayme de Altavila (FEJAL), Centro de Estudos Superiores de Maceió (CESMAC), Rua Cônego Machado 917, Farol, Cx.Postal 124, Maceió, AL 57051-160, Brasil. E-mail: flaviajabour@yahoo.com.b

${ }^{4}$ Mestranda do Curso de Pós-Graduação em Ciências Veterinárias, UFRRJ, Seropédica, RJ.
}

sementes de Jatropha curcas em caprinos, ovinos e bezerros, e com os com os frutos e as folhas de Jatropha glauca e Jatropha aceroides em caprinos, realizados por outros autores. Estas comparações indicam que, independentemente das espécies, as folhas de Jatropha spp. devem conter compostos semelhantes aos encontrados nas sementes.

TERMOS DE INDEXAÇÃO: Plantas tóxicas, intoxicação por plantas, Jatropha gossypifolia, Euphorbiaceae, ovinos, patologia.

\section{INTRODUÇÃO}

Jatropha gossypifolia L. (Euphorbiaceae) é um arbusto, encontrado em todo Brasil (Fig.1). É plantado em frente da casa de morada para "evitar a entrada de todos os males" (Lorenzi \& Matos 2002).

As sementes de Jatropha curcas são usadas como purgativo pelo homem e tem sido a causa de numerosos casos de intoxicação no homem (Watt \& Breyer-Brandwijk 1962).

Diversas outras substâncias químicas, que têm sido estudadas principalmente sob o ponto de vista estrutural ou farmacológico, foram isoladas das sementes, dos fru- 


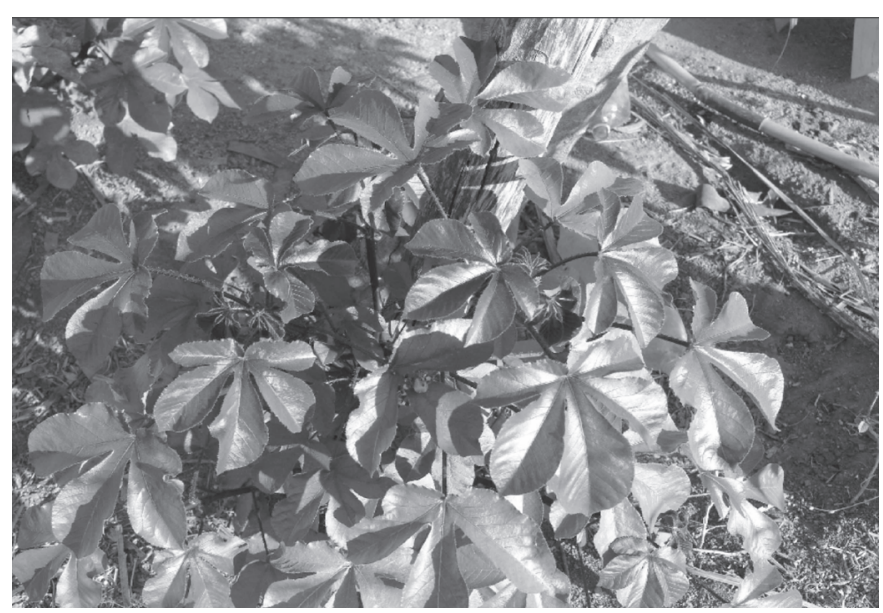

Fig.1. Jatropha gossypifolia (fam. Euphorbiaceae) no município de Seropédica, RJ.

tos, do caule, das folhas e das raízes de Jatropha spp. (Watt \& Bryer-Brandwijk 1962), inclusive de Jatropha gossypifolia (Kupchen et al. 1970, Kozhiparambil et al. 1979, Adolf et al. 1984, Horsten et al. 1996, Lorenzi \& Matos 2002, Ravindranath et al. 2003).

Com Jatropha curcas foram feitos experimentos, com as sementes, em bezerros (Ahmed \& Adam 1979a), caprinos (Adam \& Magzoub 1975, Ahmed \& Adam 1979b) e ovinos (Ahmed \& Adam 1979b), com Jatropha glauca e Jatropha aceroides, com os frutos e as folhas dessecados, em caprinos (Barri et al. 1983).

Casos naturais de intoxicação com o resíduo da extração de óleo das sementes de J. curcas foram descritos em bovinos e ovinos (Wolf 1889 apud Völker 1950, Leonhard 1889 apud Völker 1950). Torres \& Fernandes (1941) relatam que numerosos são os casos de intoxicação de animais em conseqüência de purgas com pinhão (Jatropha curcas) uma vez ou outra, seguidas de morte conforme a quantidades de sementes empregados na confecção da purga.

Não encontramos trabalhos experimentais em ruminantes sobre a ação tóxica de Jatropha gossypifolia.

Em virtude da larga distribuição de J. gossypifolia no Brasil, especialmente no Nordeste do Brasil, que mesmo em épocas de seca conserva suas folhas, estas poderiam ser ingeridas por animais com fome. $O$ objetivo deste trabalho foi verificar se as folhas de J. gossypifolia são tóxicas para ovinos, verificar a dose tóxica e estabelecer o quadro clínico-patológico, através da experimentação em ovinos. Para isto foram administradas a ovinos as foIhas frescas em diferentes doses por via oral, com o fim de obter dados que servirão como subsídio para o estabelecimento de diagnósticos em eventuais ocorrências da intoxicação sob condições naturais.

\section{MATERIAL E MÉTODOS}

Folhas de Jatropha gossypifolia (Euphorbiaceae) foram coletadas nos municípios de Seropédica e Miguel Pereira,
Estado do Rio de Janeiro, e administradas manualmente, no mesmo dia da coleta, em doses únicas de 5-40 g/ $\mathrm{kg}$, por via oral, a 13 ovinos clinicamente sadios, mestiços, machos e fêmeas, de diferentes idades, com pesos de 12,5-32 kg.

Os animais eram mantidos em baias de alvenaria, individualmente, com piso de cimento e submetidos a um jejum de 24 horas antes do início das administrações. Após a ingestão da planta recebiam capim e água à vontade e pequena quantidade de ração concentrada. Durante o experimento eram soltos na parte da manhã, durante a limpeza dos boxes, em um curral, aproximadamente por três horas. Todos os animais foram submetidos a exames clínicos, antes e, repetidas vezes, após a administração da planta, e observados até a morte ou recuperação. Foram avaliados apetite, aspecto das fezes e da urina, modificações do comportamento e da postura, cor das mucosas, temperatura, motilidade gastrintestinal, os parâmetros cardíacos e pulmonares.

Em casos de morte, era imediatamente realizada a necropsia. Fragmentos dos órgãos e tecidos eram fixados em formol a $10 \%$ e posteriormente processados, cortados em micrótomo a 5 micra e corados pela hematoxilina e eosina.

\section{RESULTADOS}

Os principais dados sobre a intoxicação experimental dos 13 ovinos que receberam as folhas frescas de Jatropha gossypifolia encontram-se no Quadro 1. Verifica-se que a dose de $40 \mathrm{~g} / \mathrm{kg}$ causou a morte de todos os ovinos e a de $5 \mathrm{~g} / \mathrm{kg}$ de nenhum; as doses intermediárias causaram a morte de parte dos animais.

O início dos sintomas após começo da ingestão da planta nos animais que morreram variou de 2 (Ov.5024) a 46 horas (Ov.5744) e a evolução da intoxicação foi de 6 dias e 6 horas (Ov.5741) a 22 dias e meio (Ov.5024). Os animais que não morreram, estavam recuperados entre 59 horas (Ov.5057) e 12 dias (5025).

Os principais sintomas foram diminuição do apetite até anorexia, fezes pastosas até líquidas, às vezes com muco e estrias de sangue, tenesmo, respiração dispnéica, na expiração com gemido, às vezes ruidosa sob forma de ronco, taquicardia, com aumento da freqüência e intensidade dos batimentos, arritmia, jugular saliente, ocasionais tremores musculares, apatia, posição com dorso arqueado, abdômen retraído, líquido escapando pela boca ou narinas, extremidades frias, emagrecimento progressivo até caquexia, dificuldade de se levantar, decúbito e morte.

À necropsia constataram-se abomaso com mucosa avermelhada (Ov.5741, 5743), com pequenas úlceras (Ov.5743, 5744), intestino vazio ou com pouco conteúdo, mucoso ou líquido (Ov.5024, 5741), a serosa do intestino avermelhada (Ov.5741, 5743), linfonodos mesentéricos aumentados, pulmões pesados com consistência de borracha (Ov. 5024, 5742, 5743, 5744), com áreas de enfisema (Ov.5741, 5744), coração flácido (Ov.5741, 5743, 5744), edema subcutâneo e coleção de líquido seroso nas cavidades torácica, pericárdica e abdominal (Ov.5026), líquido seroso na cavidade abdominal (Ov.5024).

Os exames histopatológicos revelaram no pulmão congestão (Ov.5741, 5742, 5743, 5744) e edema 
Quadro 1. Intoxicação experimental por Jatropha gossypifolia em ovinos

\begin{tabular}{|c|c|c|c|c|c|c|c|c|c|}
\hline $\begin{array}{l}\text { Ovino no. } \\
\text { (Reg. SAP) }\end{array}$ & $\begin{array}{c}\text { Peso } \\
\text { kg }\end{array}$ & $\begin{array}{c}\text { Data da } \\
\text { adminis- } \\
\text { tração da } \\
\text { planta }\end{array}$ & $\begin{array}{l}\text { Quantidade } \\
\text { da planta } \\
\text { adminis- } \\
\text { trada }\end{array}$ & $\begin{array}{r}\text { Dose } \\
\text { g/kg }\end{array}$ & Desfecho $^{a}$ & $\begin{array}{c}\text { Início dos } \\
\text { sintomas após } \\
\text { administração } \\
\text { da planta }\end{array}$ & Evolução & $\begin{array}{l}\text { Morte } \\
\text { após iní- } \\
\text { cio dos } \\
\text { sintomas }\end{array}$ & $\begin{array}{l}\text { Recuperação } \\
\text { após admi- } \\
\text { nistação da } \\
\text { planta }\end{array}$ \\
\hline 5002 & 27,5 & 15.9.92 & 137,5 & 5 & S.S. & - & - & - & - \\
\hline 5059 & 24,0 & 15.9.92 & 130,0 & 5 & S.S. & - & - & - & - \\
\hline $\begin{array}{c}5024 \\
(25772 / 74)\end{array}$ & 22,5 & 20.4 .92 & 225,0 & 10 & Morreu & $2 \mathrm{~h} 00 \mathrm{~min}$ & $\begin{array}{c}22 \text { dias } \\
14 \mathrm{~h} 22 \mathrm{~min}\end{array}$ & $\begin{array}{l}22 \text { dias } \\
16 \mathrm{~h} 25 \mathrm{~min}\end{array}$ & - \\
\hline 5057 & 13,0 & 16.9.92 & 130,0 & 10 & Sint. + & $11 \mathrm{~h} \mathrm{00min}$ & & & $59 \mathrm{~h}$ \\
\hline 5058 & 12,5 & 16.9.92 & 125,0 & 10 & Sint. + & $12 \mathrm{~h} 10 \mathrm{~min}$ & $47 \mathrm{~h} \mathrm{03min}$ & - & $59 \mathrm{~h} 13 \mathrm{~min}$ \\
\hline 5025 & 25,0 & 12.1.93 & 375,0 & 15 & Sint. ++ & $17 \mathrm{~h} 45 \mathrm{~min}$ & - & - & 12 dias $9 \mathrm{~h}$ \\
\hline 5047 & 26,0 & 13.1.93 & 390,0 & 15 & Sint. + & $31 \mathrm{~h} 40 \mathrm{~min}$ & - & - & 8 dias \\
\hline $\begin{array}{c}5026 \\
(26098 / 102)\end{array}$ & 23,0 & 3.9 .92 & 520,0 & 20 & Morreu & 19h 05min & $23 \mathrm{~h} 00 \mathrm{~min}$ & 42h 05min & - \\
\hline 5007 & 30,5 & 13.1.93 & 640,0 & 20,98 & Sint. $+(+)$ & $16 \mathrm{~h} 05 \mathrm{~min}$ & - & - & 8 dias \\
\hline $\begin{array}{c}5741 \\
(30164 / 65)\end{array}$ & 27,0 & 15.4.04 & 560,0 & 20 & Morreu & $\begin{array}{c}1 \mathrm{dia} \\
16 \mathrm{~h} 10 \mathrm{~min}\end{array}$ & $\begin{array}{c}6 \text { dias } \\
6 \mathrm{~h} 20 \mathrm{~min}\end{array}$ & $\begin{array}{c}7 \text { dias } \\
22 \mathrm{~h} 30 \mathrm{~min}\end{array}$ & - \\
\hline $\begin{array}{c}5742 \\
(30339 / 42)\end{array}$ & 32,0 & 1.7 .04 & 640,0 & 20 & Morreu & $\begin{array}{c}1 \mathrm{dia} \\
6 \mathrm{~h} 25 \mathrm{~min}\end{array}$ & $\begin{array}{l}10 \text { dias } \\
4 \mathrm{~h} 30 \mathrm{~min}\end{array}$ & $\begin{array}{c}11 \text { dias } \\
10 \mathrm{~h} 55 \mathrm{~min}\end{array}$ & - \\
\hline $\begin{array}{c}5743 \\
(30425 / 27)\end{array}$ & 24,5 & 30.11 .04 & 960,0 & 40 & Morreu & $\begin{array}{c}1 \mathrm{dia} \\
2 \mathrm{~h} 30 \mathrm{~min}\end{array}$ & $\begin{array}{c}9 \text { dias } \\
18 \mathrm{~h} 30 \mathrm{~min}\end{array}$ & 10 dias $14 \mathrm{~h}$ & - \\
\hline $\begin{array}{c}5744 \\
(30441 / 43)\end{array}$ & 27,0 & 18.1 .05 & $1.080,0$ & 40 & Morreu & $\begin{array}{c}1 \mathrm{dia} \\
22 \mathrm{~h} 40 \mathrm{~min}\end{array}$ & 7 dias & $\begin{array}{c}8 \text { dias } \\
23 \mathrm{~h} 35 \mathrm{~min}\end{array}$ & - \\
\hline
\end{tabular}

a S.S. $=$ sem sintomas, Sint. $++=$ sintomas moderados, Sint. $+=$ sintomas leves.

interlobular (Ov.5024, 5026), no coração focos de necrose incipiente (Ov.5026, 5741, 5742), no fígado leve vacuolização (Ov.5026, 5744) e necrose de hepatócitos, individual (Ov.5741) ou sob forma de pequenos focos (Ov. 5743), no tubo digestivo na mucosa áreas de congestão (Ov.5743), baço com congestão (Ov.5026, 5743, 5744).

\section{DISCUSSÃO E CONCLUSÕES}

O quadro clínico-patológico nos ovinos que receberam as folhas de Jatropha gossypifolia em nossos experimentos era caracterizado por perturbações digestivas, pulmonares, cardíacas, e ainda alterações regressivas somente evidenciadas através dos exames histológicos, em fígado e rim.

O quadro clínico-patológico observado nos experimentos com as sementes de Jatropha curcas em caprinos, ovinos e bezerros por Ahmed \& Adam (1979a,b) e Adam \& Magzoub (1975) era de diarréia, hemorragias no rúmen, retículo, pulmões, rins e coração, e alterações regressivas no fígado e rim, congestão e edema pulmonares e líquido seroso nas cavidades pré-formadas.

Esses achados são semelhantes aos nossos, com exceção das hemorragias, que não verificamos.

Em experimentos com Jatropha glauca e Jatropha aceroides em caprinos, os principais sintomas observados foram os mesmos nos animais que ingeriram os frutos e as folhas, e consistiram em diarréia, salivação, dispnéia, inapetência, depressão, consumo de água diminuído, fraqueza e emagrecimento (Barri et al. 1983).

Estas comparações indicam que as folhas devem conter, pelo menos em parte, os mesmos princípios tóxicos que as sementes, e independentemente das espécies de Jatropha.

Não temos condições de indicar os princípios tóxicos responsáveis pelo quadro clínico-patológico. Muitas substâncias foram isoladas de Jatropha spp., tanto dos frutos como das folhas e das outras partes da planta. A mais conhecida é a curcina, uma toxalbumina com ação semeIhante a ricina, crotina e ricina (Watt \& Breyer-Brandwijk 1962, Kellerman et al. 2005).

Agradecimentos.- Ao Prof. Anibal Armién, pela cessão do tema e aos Professores Marilene Brito, Paulo Vargas Peixoto e Carlos H. Tokarnia, pela orientação.

\section{REFERÊNCIAS}

Adolf W., Opferkuch H.J. \& Hecker E. 1984. Irritant phorbol derivates from four Jatropha species. Phytochemistry 23(1):129-132.

Adam S.E.I. \& Magzoub M. 1975. Toxicity of Jatropha curcas for goats. Toxicology 4:347-354.

Ahmed O.M.M. \& Adam S.E.I. 1979a. Effects of Jatropha curcas on calves. Vet. Pathol. 16:476-482.

Ahmed O.M.M. \& Adam S.E.I. 1979b. Toxicity of Jatropha curcas in sheep and goats. Res. Vet. Sci. 27:89-96.

Barri M.E.S., Onsa T.O., Elawad A.A., Elsayed N.Y., Wasfi I.A., Bari E.M.A. \& Adam S.E.I. 1983. Toxicity of five Sudanese plants to young ruminants. J. Comp. Path. 93:559-575.

Horsten S.F., can den Berag A.J., Kettenes-van den Bosch J.J., Leeflang B.R. \& Labadie R.P. 1996. Cyclogossine A: a novel cyclic heptapeptide isolated from the latex of Jatropha gossypifolia. Planta Med. 62(1):4650. PMID: 8720387 [PubMed - indexed for MEDLINE]

Kellerman T.S., Coetzer J.A.W., Naudé T.W. \& Botha C.J. 2005. Plant Poisonings and Mycotoxicoses of Livestock in Southern Africa. 2nd ed. Oxford University Press, Cape Town.

Kupchan S.M., Sigel C.W., Matz M.J., Saenz Renauld J.A., Haltiwanger 
R.C. \& Bryan R.F. 1970. Jatrophone, a novel macrocyclic diterpenoid tumor inhibitor from Jatropha gossypifolia. J. Am. Chem. Soc. 92(14):4476-4477.

Lorenzi H. \& Matos F.J.A. 2002. Plantas Medicinais no Brasil, Nativas e Exóticas. Instituto Plantarum de Estudos da Flora, Nova Odessa, SP.

Purushothaman K.K. \& Chandrasekharan S. 1979. Jatropholones A and $B$, new diterpenoids from the roots of Jatropha gossypifolia (Euphorbiaceae): crystal structure analysis of jatropholene B. Tetrahedron Letters 11:979-982.

Ravindranath N., Venkataiah B., Ramesh C., Jayaprakash P. \& Das B.
2003. Jatrophenone, a novel macrocyclic bioactive diterpene from Jatropha gossypifolia. Chem. Pharm. Bull., Tokyo, 51(7):870-871. PMID: 12843600 [PubMed, indexed for MEDLINE]

Torres S. \& Fernandes C.S. 1941. A flora de Pernambuco e a patologia animal. Arqs Inst. Pesq. Agrotécnicas, Recife, 3:35-63.

Völker R. 1950. Eugen Fröhner's Lehrbuch der Toxikologie für Tierärzte. Ferdinand Enke Verlag, Stuttgart.

Watt J.M. \& Breyer-Brandwijk M.G. 1962. The Medical and Poisonous Plants of Southern and Eastern Africa. 2nd ed. E. and S. Livingstone, Edinburgh. 